\title{
Lentes de contato esclerais: experiência inicial no Setor de Lentes de Contato da Universidade Federal de São Paulo/Escola Paulista de Medicina
}

\author{
Scleral contact lenses: first experience in the Contact Lens Sector \\ of the Universidade Federal de São Paulo/Escola Paulista de Medicina
}

\section{César Lipener ${ }^{1}$}

Fernando Leal ${ }^{2}$

\footnotetext{
${ }^{1}$ Mestre em Oftalmologia e Chefe do Setor de Lentes de Contato da Universidade Federal de São Paulo UNIFESP.

${ }^{2}$ Pós-graduando do Setor Lentes de Contato da Universidade Federal de São Paulo - UNIFESP.

Endereço para correspondência: César Lipener, Rua Manoel da Nóbrega 595 - conj. 91 - São Paulo (SP) CEP 04001-083

E-mail: lipener@uol.com.br

Recebido para publicação em 24.11.2003

Versão revisada recebida em 08.06.2004

Aprovação em 28.06.2004

Os autores não têm nenhum interesse comercial nos produtos apresentados neste estudo
}

\begin{tabular}{|l|}
\hline RESUMO \\
\hline Objetivo: Relatar a experiência inicial com o uso de lentes de contato \\
esclerais rígidas. Métodos: Estudo retrospectivo de 13 olhos de 7 pacientes \\
usuários de lentes de contato esclerais, no Setor de Lentes de Contato da \\
Universidade Federal de São Paulo/Escola Paulista de Medicina. Foram \\
coletados os seguintes dados: idade, sexo, diagnóstico, acuidades visuais \\
inicial e final, número de horas de uso diário, complicações, queixas e o \\
tempo de seguimento dos pacientes. Resultados: Foram estudados 13 \\
olhos de 7 pacientes portadores de lentes esclerais rígidas, sendo 11 lentes \\
de polimetilmetacrilato e 2 de material gás-permeável. Deste total, 6 pa- \\
cientes eram portadores de ceratocone e 1 paciente havia sido submetido \\
à ceratotomia radial. Em todos os olhos estudados houve melhora da \\
acuidade visual. Os pacientes foram estudados em média por 8 meses. \\
Conclusão: A utilização de lentes de contato esclerais pode ser uma opção \\
na clínica de lentes de contato, propiciando melhora da acuidade visual em \\
pacientes com ectasias corneanas ou submetidos à cirurgia refrativa, que \\
aguardam transplante de córnea, como também, ser uma alternativa de \\
tratamento clínico destes pacientes.
\end{tabular}

Descritores: Lentes de contato; Esclera; Acuidade visual; Transtornos da visão; Doenças da córnea; Ceratocone; Desenho de equipamento

\section{INTRODUÇ̃̃O}

As lentes de contato esclerais foram as primeiras lentes de contato a serem desenvolvidas, sendo inicialmente descritas na prática clínica por Adolf Fick ${ }^{*}$ em $1888^{(1)}$, que teve a idéia de corrigir a irregularidade da córnea com o uso de conchas esclerais de vidro que melhoravam muito a visão do paciente $^{(2)}$. A adaptação de tais lentes teve grande impulso com a introdução do polimetilmetacrilato (PMMA) em 1936, sendo a primeira lente escleral deste material produzida em $1938^{* *}$ por Obre e Muller ${ }^{(3)}$. Tais lentes foram progressivamente substituídas na contatologia moderna pelas lentes corneanas, mas adquiriram interesse renovado com o desenvolvimento das lentes de contato esclerais rígidas-gás-permeáveis ${ }^{(4-5)}$.

$\mathrm{O}$ advento das lentes de contato esclerais rígidas-gás-permeáveis (RGP) reduziu as complicações decorrentes da hipóxia, quando comparadas com

\footnotetext{
Adolf Fick 1888 apud $^{(1)}$

** Obre e Muller 1938 apud $^{(3)}$
} 
as lentes esclerais de PMMA para uso diário, dando uma nova dimensão ao potencial de utilização de lentes esclerais ${ }^{(3,5)}$.

A adaptação das lentes tocando a esclera elimina a necessidade do contato com a córnea, oferecendo as seguintes vantagens $^{(6)}$ :

- Adaptação em córneas com topografias irregulares.

- Maior estabilidade para lentes com alto poder dióptrico.

- Menor sensação palpebral.

- Menor retenção de corpos estranhos atrás das lentes.

- Menor perda das lentes, devido à ação palpebral.

- Utilização mais fácil em pacientes menos habilidosos e com dificuldades visuais como, por exemplo, afácicos.

Entre as desvantagens das lentes esclerais podemos citar:

- Produção mais dispendiosa quando comparada com outros tipos de lentes.

- Seu tamanho pode intimidar alguns pacientes, provocando sensação de volume e aparência de pseudo-proptose.

- As fenestrações das lentes podem deixar passar bolhas de ar para o fluido pré-corneano, provocando alterações visuais.

- As fenestrações também podem produzir um gradiente de pressão no fluido pré-corneano, levando a uma adaptação mais apertada, especialmente no limbo.

- Hipóxia, principalmente com as lentes de PMMA.

Este estudo tem como objetivo relatar a experiência inicial obtida no Setor de Lentes de Contato da Escola Paulista de Medicina com a adaptação de lentes de contato esclerais rígidas com fins visuais.

\section{MÉTODOS}

Foi realizado um estudo retrospectivo de 7 pacientes (total de 13 olhos) usuários de lentes de contato esclerais rígidas (Lentes Froillens ${ }^{\circledR}$ ), no Setor de Lentes de Contato da Universidade Federal de São Paulo/Escola Paulista de Medicina, no período de 2000 a 2003. Foram coletados os seguintes dados: idade e sexo dos pacientes, diagnóstico, acuidades visuais inicial e final, número de horas de uso diário, complicações e tempo de seguimento dos pacientes.

\section{RESULTADOS}

A idade dos pacientes variou de 10 a 58 anos. Dos sete casos relatados, 5 eram do sexo masculino e 2 do sexo feminino. Com relação ao diagnóstico seis pacientes eram portadores de ceratocone e 1 foi submetido a ceratotomia radial.Todos os pacientes estudados apresentaram melhora importante da acuidade visual. Dos treze olhos estudados, todos possuíam acuidade visual inicial menor ou igual a 20/100, sendo que em oito olhos a acuidade visual final foi igual ou superior a 20/50 . O número de horas de uso diário foi obtido em 5 pacientes, num total de 9 olhos. Destes, 3 pacientes faziam uso da lente durante 3 a 4 horas por dia (Tabela 1).
Em 6 pacientes foi possível obtermos dados referentes à adaptação com lentes de contato corneanas RGP: em 2 deles o insucesso foi decorrente de intolerância e mobilidade excessiva, em 2 devido à mobilidade excessiva e em 2 a descentração (Figura 1).

Foram observadas complicações e/ou queixas em um total de 4 olhos: desconforto em 1, desconforto e neovascularização em um, e neovascularização em 2 olhos (Tabela 1). Neste estudo, um dos pacientes, com história de ceratotomia radial prévia, apresentou aumento de neovascularização corneana com o uso de lentes esclerais rígidas-gás-permeáveis.

\section{DISCUSSÃO}

Em um estudo retrospectivo sobre uso de lentes de contato esclerais rígidas-gás-permeáveis em 530 pacientes, a faixa etária variou de 2 a 94 anos, sendo que 337 eram do sexo masculino e 193 do sexo feminino(7). Em outro estudo com 44 casos, também retrospectivo, a idade dos pacientes estudados variou de 15 a 71 anos, sendo 28 pacientes do sexo masculino e 16 do sexo feminino. Neste trabalho, dos sete pacientes estudados 4 eram do sexo masculino e dois do sexo feminino, com idades variando de 10 a 58 anos.

As indicações do uso de contato de lentes esclerais são: melhorar a acuidade visual (indicação visual); melhorar a superfície ocular e reduzir o desconforto em doenças oculares, como por exemplo, a síndrome de Stevens-Johnson (indicação terapêutica) e melhorar a aparência do olho, como por exemplo, em traumas e phthisis bulbi (indicação cosmética) ${ }^{(1)}$. Dos sete pacientes apresentados neste trabalho, 6 eram portado-

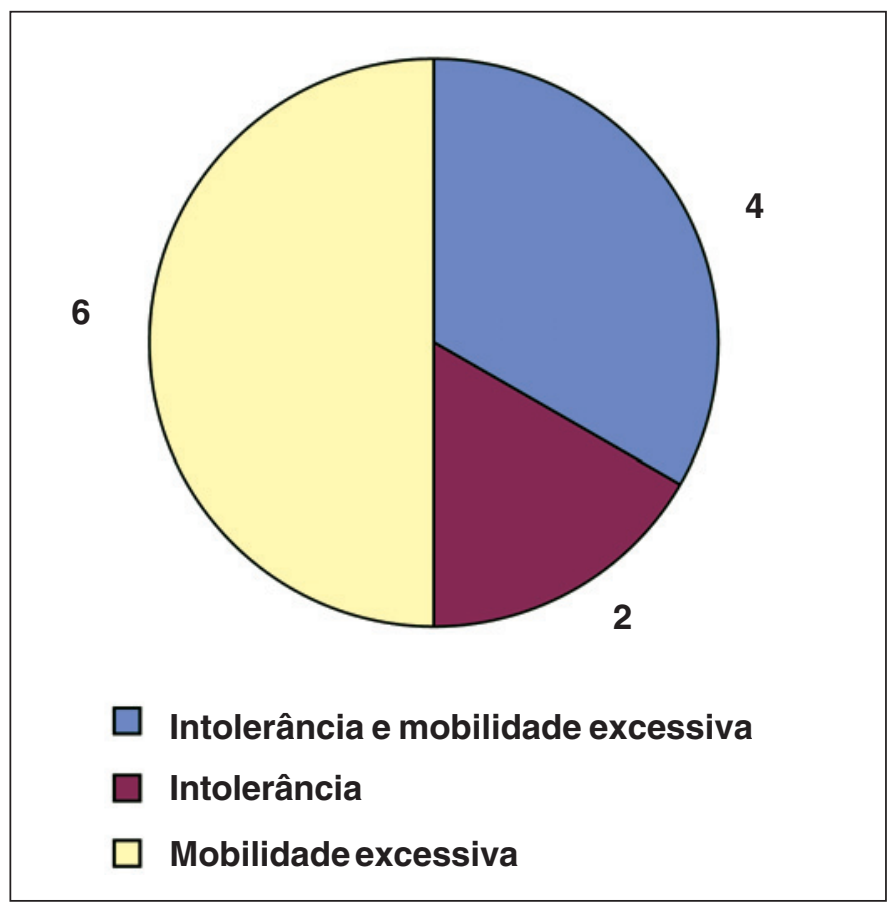

Figura 1 - Causas de insucesso na adaptação de lentes corneanas RGP em 12 olhos 


\begin{tabular}{|c|c|c|c|c|c|c|c|c|c|}
\hline № & Sexo & Idade & Olho & $\begin{array}{c}\text { AV } \\
\text { inicial }\end{array}$ & $\begin{array}{l}\text { AV } \\
\text { final }\end{array}$ & $\begin{array}{l}\text { Horas de } \\
\text { uso diário }\end{array}$ & $\begin{array}{l}\text { Complicações } \\
\text { e/ou queixas }\end{array}$ & $\begin{array}{c}\text { Tempo de } \\
\text { seguimento (meses) }\end{array}$ & Material \\
\hline 1 & M & 10 & $\begin{array}{l}\text { OD } \\
\text { OE }\end{array}$ & $\begin{array}{l}20 / 100 \\
20 / 400\end{array}$ & $\begin{array}{l}20 / 50 \\
20 / 50\end{array}$ & $\begin{array}{l}3 \\
4\end{array}$ & Desconf. & 2 & $\begin{array}{l}\text { PMMA } \\
\text { PMMA }\end{array}$ \\
\hline 2 & M & 12 & OD & $20 / 400$ & $20 / 30$ & 7 & - & 6 & PMMA \\
\hline \multirow[t]{2}{*}{3} & $\mathrm{~F}$ & 31 & OD & $\mathrm{CD} 30 \mathrm{~cm}$ & $20 / 60$ & - & - & 2 & PMMA \\
\hline & & & OE & $20 / 400$ & $20 / 50$ & - & & & PMMA \\
\hline 4 & $\mathrm{~F}$ & 30 & $\begin{array}{l}\text { OD } \\
\text { OE }\end{array}$ & $\begin{array}{l}20 / 100 \\
20 / 100\end{array}$ & $\begin{array}{l}20 / 25 \\
20 / 30\end{array}$ & - & - & 2 & $\begin{array}{l}\text { PMMA } \\
\text { PMMA }\end{array}$ \\
\hline 5 & M & 58 & $\begin{array}{l}\text { OD } \\
\text { OE }\end{array}$ & $\begin{array}{l}20 / 400 \\
20 / 400\end{array}$ & $\begin{array}{l}20 / 100 \\
20 / 30\end{array}$ & $\begin{array}{l}4 \\
8\end{array}$ & Neovasc./Desconf. & 24 & $\begin{array}{l}\text { PMMA } \\
\text { PMMA }\end{array}$ \\
\hline 6 & M & 11 & $\begin{array}{l}\text { OD } \\
\text { OE }\end{array}$ & $\begin{array}{l}\text { CD } 2 \mathrm{~m} \\
\mathrm{CD} 2 \mathrm{~m}\end{array}$ & $\begin{array}{l}20 / 100 \\
20 / 100\end{array}$ & $\begin{array}{l}8 \\
8\end{array}$ & - & 12 & $\begin{array}{l}\text { PMMA } \\
\text { PMMA }\end{array}$ \\
\hline 7 & M & 56 & $\begin{array}{l}\text { OD } \\
\text { OE }\end{array}$ & $\begin{array}{l}20 / 400 \\
20 / 400\end{array}$ & $\begin{array}{l}20 / 70 \\
20 / 30\end{array}$ & $\begin{array}{l}14 \\
14\end{array}$ & $\begin{array}{l}\text { neovasc. } \\
\text { neovasc. }\end{array}$ & 8 & $\begin{array}{l}\text { RGP } \\
\text { RGP }\end{array}$ \\
\hline
\end{tabular}

res de ceratocone e 1 foi submetido a ceratotomia radial, tendo portando indicação visual. A literatura relata uma proporção de indicação visual de adaptação de lentes esclerais de $80,3 \%$ e de indicações terapêuticas de $16,8 \%{ }^{(6)}$.

As principais indicações visuais de adaptação das lentes esclerais são as ectasias corneanas primárias, incluindo ceratocone, ceratoglobo e a degeneração marginal pelúcida. Outras indicações visuais importantes são adaptações pós-transplante de córnea, afacia e altas miopias. Os pacientes com indicação de adaptação visual, em geral, são os que obtêm maior benefício com as lentes esclerais, devido aos seguintes fatores: a criação de um filme lacrimal entre o olho e superfície posterior da lente, capaz de corrigir defeitos ópticos decorrentes de astigmatismos corneanos irregulares e maior estabilidade de lentes com altos poderes refrativos ${ }^{(1)}$.

Segundo a literatura, as indicações do uso terapêutico de lentes esclerais decorrem da sua capacidade de reter lágrima em condições de olho seco severo e proteção ocular no caso de ceratite de exposição e anormalidade de cílios e pálpebras. A principal indicação é na síndrome de Stevens-Johnson e em

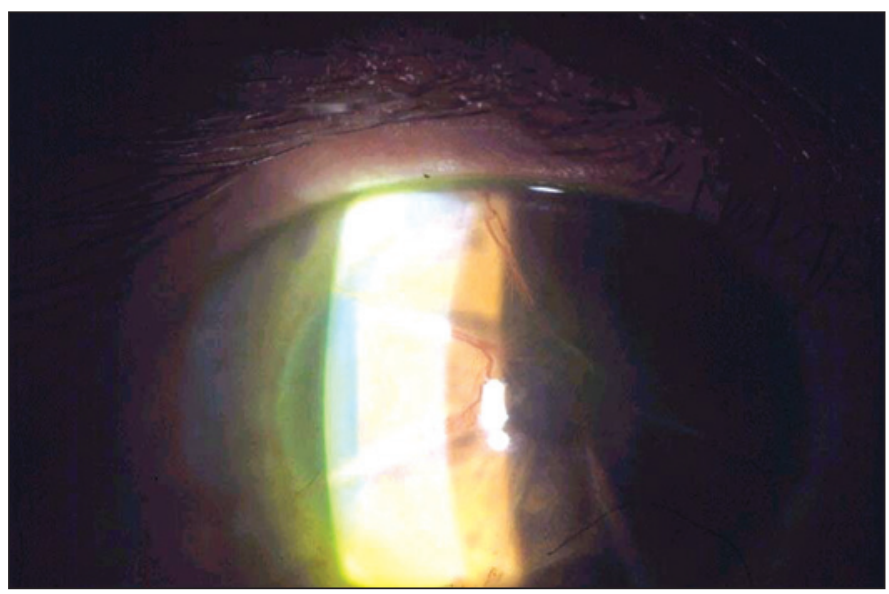

Figura 2 - Neovascularização em paciente com ceratotomia radial prévia e usuário de lente escleral RGP outras doenças tais como: penfigóide ocular cicatricial, ceratopatia de exposição, ceratite pós-herpética, deficiência congênita das glândulas de Meibomius, ceratoconjuntivite límbica superior e síndrome de Sjögren ${ }^{(8-9)}$.

Os sete pacientes estudados apresentaram melhora importante da acuidade visual, sendo que 10 destes $(76,92 \%)$ ficaram com visão igual ou superior a 20/70. Em um estudo com 530 pacientes, $77 \%$ destes alcançaram visão superior a 20/70 e $49 \%$ visão superior a $20 / 30^{(7)}$. Em pacientes portadores de ectasias corneanas primárias o transplante corneano permanece como uma importante opção terapêutica, especialmente em pacientes com visão inferior a $20 / 40^{(7)}$. Neste caso a utilização de lentes esclerais pode propiciar aumento da acuidade visual em pacientes na fila para transplante de córnea, sendo uma alternativa de tratamento conservador. Segundo a literatura alguns pacientes preferem usar as lentes esclerais e não serem submetidos ao transplante, mesmo com o potencial de melhora de acuidade visual que o tratamento cirúrgico pode oferecer ${ }^{(7)}$.

Entre os pacientes estudados, o número de horas de uso diário pode ser obtido em 5 pacientes, total de 9 olhos. Destes, em 3 olhos, os pacientes faziam uso da lente durante 3 a 4 horas por dia. Estudos relatam variação no uso de horas por dia de 3 a 24 horas, com média de 8,5 horas, em pacientes com indicações visuais de adaptação de lentes esclerais ${ }^{(1)}$.

Segundo a literatura ${ }^{(7)}$ as principais complicações associadas ao uso de lentes de contato esclerais são edema corneano $(27 \%)$, desconforto $(24 \%)$, neovascularização corneana central (14\%) e neovascularização corneana periférica (12\%). Foram observadas complicações e/ou queixas em um total de 5 olhos: desconforto em 2 olhos, desconforto e neovascularização em um olho e neovascularização em 2 olhos. Dentre os pacientes estudados um apresentou neovascularização importante mesmo com o uso de lentes esclerais rígidas-gáspermeáveis, adaptadas após o paciente ter sido submetido a ceratotomia radial. A literatura cita que a neovascularização corneana após ceratotomia radial, quando presente, está sem- 
pre localizada superficialmente dentro das incisões e parece não ter relação com a sua extensão, mas sim com a distância das incisões do limbo vascular ${ }^{(10)}$. Segundo a literatura ${ }^{(3,11)}$ a introdução de lentes esclerais RGP em substituição as lentes esclerais de PMMA, provocou grande redução nas complicações devidas a hipóxia corneana.

Em 6 pacientes foi possível obter dados referentes à adaptação com lentes de contato corneanas RGP, sendo que em 2 pacientes o insucesso foi decorrente de intolerância e mobilidade excessiva, em 2 devido à mobilidade excessiva e em 2 a descentração. Estes dados estão de acordo com os relatos de que a maioria dos pacientes usuários de lentes esclerais tentaram adaptação ou foram usuários de lentes corneanas, e que as principais causas de insucesso com estas últimas são o desconforto e a grande mobilidade ${ }^{(7)}$.

\section{CONCLUSÃO}

A utilização de lentes de contato esclerais é uma opção na clínica de lentes de contato, propiciando melhora da acuidade visual em pacientes com doenças corneanas que aguardam transplante, sendo uma alternativa não cirúrgica para o tratamento dos mesmos.

\section{ABSTRACT}

Purpose: To describe initial experiences with patients wearing rigid escleral contact lenses. Methods: Retrospective study of 13 eyes of 7 patients, 11 fitted with polymethylmethacrylate and two with rigid gas-permeable scleral contact lenses. Age, sex, diagnosis, initial and final visual acuity (VA), time of daily wear, complications and follow-up were recorded. Results:
Keratoconus was diagnosed in 6 patients, one was submitted to radial keratotomy. Mean follow-up lasted 8 months. Visual acuity of all eyes improves with scleral contact lenses. Conclusion: Visual acuity improvement observed in corneal ectasia and after radial keratotomy, in patients awaiting corneal graft, suggests that scleral contact lenses can be considered clinical treatment alternative.

Keywords: Contact lenses; Sclera; Visual acuity; Vision disorders; Corneal diseases; Keratoconus; Equipment design

\section{REFERÊNCIAS}

1. Foss AJ, Trodd TC, Dart JK. Current indications for scleral contact lens. CLAO J. 1994;20(2):115-8.

2. Pena AS. Breve histórico das lentes de contato. In: Pena AS, editor. Clínica de lente de contato. Rio de Janeiro: Cultura Médica; 1989. p.1-2.

3. Giovedi Filho R, Giovedi MR. Materiais, classificação e forma de uso das lentes de contato. In: Coral-Ghanem C, Kara José N, editor. Lentes de contato. Rio de Janeiro: Cultura Médica; 2003. p.1-2.

4. Ruben CM, Benjamin WJ. Scleral contact lenses: preliminary report on oxygen-permeable materials. Contact Lens. 1985;13:5-10.

5. Bleshoy H, Pullum KW. Corneal reponse to gas permeable impression scleral lenses. J Br Contact Lens Assoc.1998;11;31-7

6. Tan DT, Pullum KW, Buckley RJ. Medical aplications of scleral contact lenses: gás-permeable scleral contact lenses. Cornea. 1995;14(2):130-7.

7. Pullum KW, Buckley RJ. A study of 530 patients referred for rigid gas permeable scleral contact lens assessment. Cornea. 1997;16(6):612-22.

8. Romero-Rangel T, Stavrou P, Cotter J, Rosenthal P, Baltatzis S, Foster CS. Gas-permeable scleral contact lens terapy in ocular surface disease. Am J Ophhthalmol. 2000;130(1):25-32.

9. Cotter JM, Rosenthal P. Scleral contact lenses. J Am Optom Assoc. 1998; 69(1):33-40.

10. Moreira S, Moreira H. Adaptação de lentes de contato após ceratotomia radial. In: Moreira S, Moreira H, editor. Lentes de contato. Rio de Janeiro: Cultura Médica; 1998. p.238-9.

11. Pullum KW, Hobley AJ, Parker JH. Hypoxic corneal changes following sealed gas permeable impression scleral lens wear. J Br Contact Lens Assoc. 1990;13:83-7. 